\title{
Accurately and Efficiently Interpreting Human-Robot Instructions of Varying Granularities
}

\author{
Dilip Arumugam*, Siddharth Karamcheti*, Nakul Gopalan, Lawson L.S. Wong, and Stefanie Tellex \\ Computer Science Department, Brown University, Providence, RI 02912 \\ \{dilip_arumugam@, siddharth_karamcheti@,ngopalan@cs., 1sw@, stefie10@cs.\}brown.edu \\ * The first two authors contributed equally
}

\begin{abstract}
Humans can ground natural language commands to tasks at both abstract and fine-grained levels of specificity. For instance, a human forklift operator can be instructed to perform a high-level action, like "grab a pallet" or a low-level action like "tilt back a little bit." While robots are also capable of grounding language commands to tasks, previous methods implicitly assume that all commands and tasks reside at a single, fixed level of abstraction. Additionally, methods that do not use multiple levels of abstraction encounter inefficient planning and execution times as they solve tasks at a single level of abstraction with large, intractable state-action spaces closely resembling real world complexity. In this work, by grounding commands to all the tasks or subtasks available in a hierarchical planning framework, we arrive at a model capable of interpreting language at multiple levels of specificity ranging from coarse to more granular. We show that the accuracy of the grounding procedure is improved when simultaneously inferring the degree of abstraction in language used to communicate the task. Leveraging hierarchy also improves efficiency: our proposed approach enables a robot to respond to a command within one second on $90 \%$ of our tasks, while baselines take over twenty seconds on half the tasks. Finally, we demonstrate that a real, physical robot can ground commands at multiple levels of abstraction allowing it to efficiently plan different subtasks within the same planning hierarchy.
\end{abstract}

\section{INTRODUCTION}

In everyday speech, humans use language at multiple levels of abstraction. For example, a brief transcript from an expert human forklift operator instructing a human trainee has very abstract commands such as "Grab a pallet," mid-level commands such as "Make sure your forks are centered," and very fine-grained commands such as "Tilt back a little bit" all within thirty seconds of dialog. Humans use these varied granularities to specify and reason about a large variety of tasks with a wide range of difficulties. Furthermore, these abstractions in language map to subgoals that are useful when interpreting and executing a task. In the case of the forklift trainee above, the sub-goals of moving to the pallet, placing the forks under the object, then lifting it up are all implicitly encoded in the command "Grab a pallet." By decomposing generic, abstract commands into modular sub-goals, humans exert more organization, efficiency, and control in their planning and execution of tasks. A robotic system that can identify and leverage the degree of specificity used to communicate instructions would be more accurate in its task grounding and more robust towards varied human communication.

Existing approaches map between natural language commands and a formal representation at some fixed level of

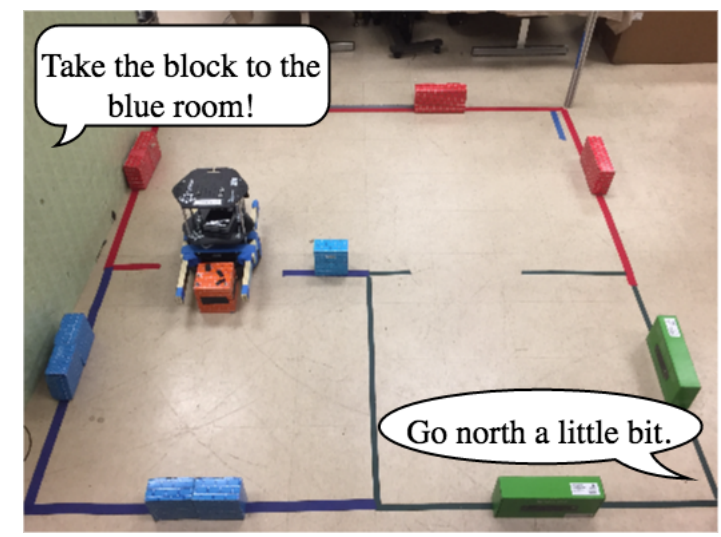

Fig. 1: Examples of high-level and fine-grained commands issued to the Turtlebot robot in a mobile-manipulation task.

abstraction [8, 22, 34]. While effective at directing robots to complete predefined tasks, mapping to fixed sequences of robot actions is unreliable in changing or stochastic environments. Accordingly, MacGlashan et al. [20] decouple the problem and use a statistical language model to map between language and robot goals, expressed as reward functions in a Markov Decision Process (MDP). Then, an arbitrary planner solves the MDP, resolving any environment-specific challenges with execution. As a result, the learned language model can transfer to other robots with different action sets so long as there is consistency in the task representation (i.e., reward functions). However, MDPs for complex, real-world environments face an inherent tradeoff between including low-level task representations and increasing the time needed to plan in the presence of both low- and high-level reward functions [14].

To address these problems, we present an approach for mapping natural language commands of varying complexities to reward functions at different levels of abstraction within a hierarchical planning framework. This approach enables the system to quickly and accurately interpret both abstract and fine-grained commands. Our system uses a deep neural network language model that maps natural language commands to the appropriate level of the planning hierarchy. By coupling abstraction-level inference with the overall grounding problem, we exploit the subsequent hierarchical planner to efficiently execute the grounded tasks. To our knowledge, we are the first to contribute a system for grounding language at multiple levels of abstraction, as well as the first to contribute a deep 
learning system for improved robotic language understanding. Our evaluation shows that deep neural network language models can infer reward functions more accurately than statistical language model baselines. We present results comparing a traditional statistical language model to three different neural architectures that are commonly used in natural language processing. Furthermore, we show that a hierarchical approach allows the planner to map to a larger, richer space of reward functions more quickly and more accurately than non-hierarchical baselines. This speedup allows the robot to respond faster and more accurately to a user's request, with a much larger set of potential commands than previous approaches. We also demonstrate on a Turtlebot the rapid and accurate response of our system to natural language commands at varying levels of abstraction.

\section{RELATED WORK}

Humans use natural language to communicate ideas, motivations, task descriptions, etc. with other humans. Some of the earliest works in this area mapped tasks to another planning language, which then grounded to the actions performed by the robots [13, 8]. More recent methods ground natural language commands to tasks using features that describe correspondences between natural language phrases present in the task description to the physical objects [15, 22, 34, 5, 30], or abstract spatial concepts [28], present in the world and the actions available in the world. This featurized representation can then describe the sequence of actions needed to complete the task. All these approaches ground commands to action sequences, leading to brittle behavior if the environment is stochastic.

MacGlashan et al. [20] proposed grounding natural language commands to reward functions associated with certain tasks, allowing robot agents to plan in stochastic environments. They treat the goal reward function as a sequence of propositional functions, much like a machine language, to which a natural language task can be translated, using an IBM Model 2 [6, 7] (IBM2) language model. While their propositional functions only lie at one level of abstraction, we want the robot to understand commands at different levels of specificity while still maintaining efficient planning and execution in the face of multiple levels of abstraction.

Crucially, MacGlashan et al. [20] actually perform inference over reward function templates, or lifted reward functions, along with environmental constraints. A lifted reward function merely specifies a task while leaving the environment-specific variables of the task undefined. The environmental binding constraints then specify the properties that an object in the environment must satisfy in order to be bound to a lifted reward function variable. By doing this, the output space of the language model is never tied to any particular instantiation of the environment, but can instead align to objects and attributes that lie within some distribution over environments. Given a lifted reward function and environment constraints (henceforth jointly referred to as only a lifted reward function), a subsequent model can later infer the environment-specific variables without needing to relearn the language understanding components for each environment. In order to leverage this flexibility, all of our proposed language models produce lifted reward functions which are then completed by a grounding module before being passed to the planner (see Sec. IV).

Planning in domains with large state-action spaces is computationally expensive as planners like value iteration and bounded real-time dynamic programming (RTDP) need to explore the domain at the lowest, "flat" level of abstraction [3, 24]. Naively this might result in an exhaustive search of the space before the goal state is found. A better approach is to decompose the planning problem into smaller, more easily solved subtasks. The agent can then achieve the goal by choosing a sequence of these subtasks. A common method to describe subtasks is by using temporal abstraction in the form of macro-actions [23] or options [33]. These methods achieve subgoals using either a fixed sequence of actions [23] or a subgoal based policy [33]. Planning with macro-actions or options requires computing the policies for each option or macro-action, which is done by exploring and backing up rewards from lowest level actions. This "bottom-up" planning is slow, as the reward for each action taken needs to be backed up through the hierarchy of options, which is time consuming. Other methods for abstraction, like MAXQ [11], R-MAXQ [17] and Abstract Markov Decision Processes (AMDPs) [14] involve providing a hierarchy of subtasks. In these methods, a subtask is associated with a subgoal and a state abstraction relevant to achieving the subgoal [11, 14, 17]. Both MAXQ [11] and R-MAXQ [17] are bottom-up planners, they back up each individual action's reward across the hierarchy.

We use AMDPs [14] in this paper because they plan in a "top-down" fashion. AMDPs offer model-based hierarchical representations in the form of reward functions and transition functions to every subtask. An AMDP hierarchy itself is an acyclic graph in which each node is a primitive action or an AMDP that solves a subtask defined by its parent; the states of each subtask AMDP are abstract representations of the environment state. AMDPs have been shown to achieve faster planning performance than other hierarchical methods [14].

We use a deep neural network language model to perform language grounding. Deep neural networks have had great success in many natural language processing (NLP) tasks, such as traditional language modeling [4, 25, 26], machine translation [9, 10], and text categorization [16]. One reason for their success is the ability to learn meaningful input representations [4, 27]. These "embeddings" are dense vectors that not only uniquely represent individual words (as opposed to otherwise sparse approaches for word representation), but also capture semantically significant features of the language. Another reason is the use of recurrent neural networks (RNNs), a type of neural network cell that maps variable length inputs (i.e. commands) to a fixed-size vector representation, which have been widely used in NLP [9, 10, 35]. Our approach uses both word embeddings and a state-of-the-art RNN model to map between natural language and MDP reward functions. 


\section{TECHNICAL APPROACH}

To interpret a variety of natural language commands, there must be a representation for all possible tasks and subtasks. We specify an Object-oriented Markov Decision Process (OOMDP) to model the robot's environment and actions [12]. An MDP is a five-tuple of $\langle\mathcal{S}, \mathcal{A}, \mathcal{T}, \mathcal{R}, \gamma\rangle$ where $\mathcal{S}$ represents the set of states that define an environment, $\mathcal{A}$ denotes the set of actions an agent can execute to transition between states, $\mathcal{T}$ defines the transition probability distribution over all possible next states given a current state and executed action, $\mathcal{R}$ defines the numerical reward earned for a particular transition, and $\gamma$ represents the discount factor or effective time horizon under consideration. Planning in an MDP produces a mapping between states and actions, or policy, that maximizes the total expected discounted reward. In our framework, as in MacGlashan et al. [20], we will map between words in language and specific reward functions.

An OO-MDP builds upon an MDP by adding sets of object classes and propositional functions; each object class is defined by a set of attributes and each propositional function is parameterized by instances of object classes. For example, an OO-MDP for the mobile robot manipulation domain seen in Fig. 1 might denote the robot's successful placement of the orange block into the blue room via the propositional function blockInRoom block0 room1, where block0 and room 1 are instances of the block and room object classes respectively and the blockInRoom propositional function checks if the location attribute of block 0 is contained in room 1 . Using these propositional functions as reward functions that encode termination conditions for each task, we arrive at a sufficient, semantic representation for grounding language. For our evaluation, we use the Cleanup World [18, 20] OO-MDP, which models a mobile manipulator robot; this domain is defined in Sec. V-A

However, this approach does not generalize well to different environment configurations. At training time, any natural language command that moves objects or agents to a specific room is conditioned to map room attributes to specific room instances (i.e. in the case of Fig. 1, the blue room is always room1). With this in mind, consider what happens if we switched the blue and green rooms at test time, so that the green room is now room1. In this case, any language command that moves an object or agent to the blue room would fail, as the room instances have been switched around.

To this end, we "lift" the propositional functions from before, to better generalize to unseen environments. Given a command like "Take the block to blue room," the corresponding lifted propositional function takes the form blocklnRoom block0 roomlsBlue, denoting that the block should end up in the room that is blue. We then assume an environment-specific grounding module (see Sec. IV-C) that consumes these lifted reward functions and performs the actual low-level binding to specific room instances, which can then be passed to a planner.

In order to effectively ground commands across multiple levels of complexity, we assume a predefined hierarchy over the state-action space of the given grounding environment.
Furthermore, each level of this hierarchy requires its own set of reward functions for all relevant tasks and sub-tasks. In our work, fast planning and the ability to ground and solve individual subtasks without needing to solve the entire planning problem make AMDPs a reliable choice for the hierarchical planner [14]. Finally, we assume that all commands are generated from a single, fixed level of abstraction.

Given a natural language command $c$, we find the corresponding level of the abstraction hierarchy $l$, and the lifted reward function $m$ that maximizes the joint probability of $l$, $m$ given $c$. Concretely, we seek the level of the state-action hierarchy $\hat{l}$ and the lifted reward function $\hat{m}$ such that:

$$
\hat{l}, \hat{m}=\arg \max _{l, m} \operatorname{Pr}(l, m \mid c)
$$

For example, as illustrated in Fig. 1, a high-level natural language command like "Take the block to the blue room" would map to the highest abstraction level, while a low-level command like "Go north a little bit" would map to the finestgrained level. We estimate this joint probability by learning a language model (described in Sec. IV] and training on a parallel corpus that pairs natural language commands with a corresponding reward function at a particular level of the abstraction hierarchy.

Given this parallel corpus, we train each model by directly maximizing the joint probability from Eqn. 11. Specifically, we learn parameters $\hat{\theta}$ that maximize the corpus likelihood:

$$
\hat{\theta}=\arg \max _{\theta} \prod_{(c, l, m) \in \mathbb{C}} \operatorname{Pr}(l, m \mid c, \theta)
$$

At inference time, given a language command $c$, we find the best $l, m$ that maximize the probability $\operatorname{Pr}(l, m \mid c, \hat{\theta})$. The lifted reward function $m$ is then completed by the grounding module (see Sec. IV-C) and passed to a hierarchical planner, which plans the corresponding task at abstraction level $l$.

\section{Language Models}

We compare four language models: an IBM Model 2 translation model (similar to MacGlashan et al. [20]), a deep neural network bag-of-words language model, and two recurrent neural network (RNN) language models, with varying architectures. For detailed descriptions and implementations of all the presented models, as well as the datasets used throughout this paper, please refer to the supplemental repository:

https://github.com/h2r/GLAMDP

\section{A. IBM Model 2}

As a baseline, task grounding is formulated as a machine translation problem, with natural language as the source language and semantic task representations (lifted reward functions) as the target language. We use the well-known IBM Model 2 (IBM2) machine translation model [6, 7] as a statistical language model for scoring reward functions given input commands. IBM2 is a generative model that solves the following objective (equivalent to Eqn. 1 by Bayes' rule):

$$
\hat{l}, \hat{m}=\arg \max _{l, m} \operatorname{Pr}(l, m) \cdot \operatorname{Pr}(c \mid l, m)
$$




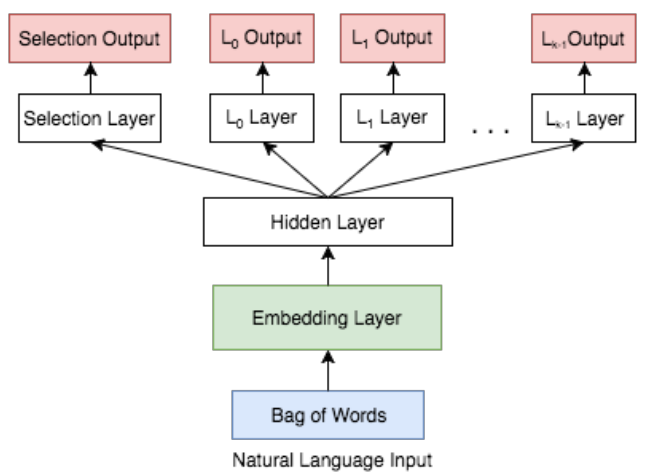

(a) Multi-NN Model

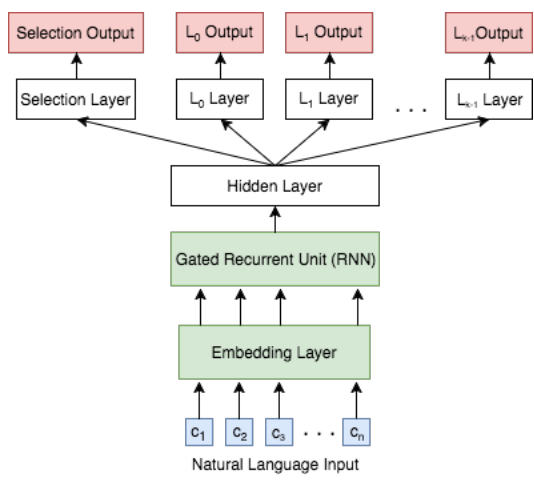

(b) Multi-RNN Model

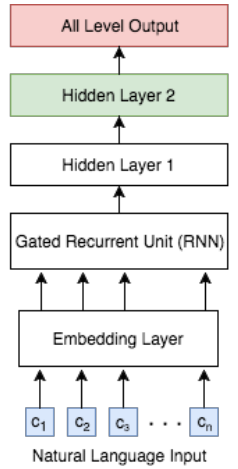

(c) Single-RNN Model

Fig. 2: Model architectures for all three sets of deep neural network models. In blue are the network inputs, and in red are the network outputs. Going left to right, the green denotes significant structural differences between models.

This task grounding formulation follows directly from MacGlashan et al. [20] and we continue in an identical fashion training the IBM2 using the standard EM algorithm.

\section{B. Neural Network Language Models}

We develop three classes of neural network architectures (see Fig. 2): a feed-forward network that takes a natural language command encoded as a bag-of-words and has separate parameters for each level of abstraction (Multi-NN), a recurrent network that takes into account the order of words in the sequence, also with separate parameters (Multi-RNN), and a recurrent network that takes into account the order of words in the sequence and has a shared parameter space across levels of abstraction (Single-RNN).

1) Multi-NN: Multiple Output Feed-Forward Network: We propose a feed-forward neural network [4, 16, 27] that takes in a natural language command $c$ as a bag-of-words vector $\vec{c}$, and outputs both the probability of each of the different levels of abstraction, as well as the probability of each reward function. We decompose the conditional probability from Eqn. 1 as $\operatorname{Pr}(l, m \mid c)=\operatorname{Pr}(l \mid c) \cdot \operatorname{Pr}(m \mid l, c)$. Applying this to the corpus likelihood (Eqn. 2) and taking logarithms, the Multi-NN objective is to find parameters $\hat{\theta}$ :

$$
\hat{\theta}=\arg \max _{\theta} \sum_{(\vec{c}, l, m)} \log \operatorname{Pr}(l \mid \vec{c}, \theta)+\log \operatorname{Pr}(m \mid l, \vec{c}, \theta)
$$

To learn this set of parameters, we use the architecture shown in Fig. 2a Namely, we employ a multi-output deep neural network with an initial embedding layer, a hidden layer that is shared between each of the different outputs, and then output-specific hidden and read-out layers, respectively.

The level selection output is a $k$-element discrete distribution, where $k$ is the number of levels of abstraction in the given planning hierarchy. Similarly, the reward function output at each level $L_{i}$ is an $r_{i}$-element distribution, where $r_{i}$ is the number of reward functions at the given level of the hierarchy.

To train the model, we minimize the sum of the crossentropy loss on each term in Eqn. 4. We train the network via backpropagation, using the Adam Optimizer [19], with a minibatch size of 16 , and a learning rate of 0.001 . Furthermore, to better regularize the model and encourage robustness, we use Dropout [31] after the initial embedding layer, as well as after the output-specific hidden layers with probability $p=0.5$.

2) Multi-RNN: Multiple Output Recurrent Network: Inspired by the success of recurrent neural networks (RNNs) in NLP tasks [9, 25, 26, 32], we propose an RNN language model that takes in a command as a sequence of words and, like the Multi-NN bag-of-words model, outputs both the probability of each of the different levels of abstraction, as well as the probability of each reward function, at each level of abstraction. RNNs extend feed-forward networks to handle variable length inputs by employing a set of one or more hidden states, which are updated after reading in each input token. Instead of converting natural language command $c$ to a vector $\vec{c}$, we use an RNN to interpret it as a sequence of words $s=\left\langle c_{1}, c_{2} \ldots c_{n}\right\rangle$. The Multi-RNN objective is then:

$$
\hat{\theta}=\arg \max _{\theta} \sum_{(c, l, m)} \log \operatorname{Pr}(l \mid s, \theta)+\log \operatorname{Pr}(m \mid l, s, \theta)
$$

This modification is reflected in Fig. 2b which is similar to the Multi-NN architecture, except in the lower layers where we use an RNN encoder that takes the sequence of raw input tokens and maps them into a fixed-size state vector. We use the gated recurrent unit (GRU) of Cho et al. [9], a particular type of RNN cell that have been shown to work well on natural language sequence modeling tasks [10].

Similar to the Multi-NN, we train the model by minimizing the sum of the cross-entropy loss of each of the two terms in Eqn. 5, with the same optimizer setup as the Multi-NN model. Dropout is used to regularize the network after the initial embedding layer and the output-specific hidden layers.

3) Single-RNN: Single Output Recurrent Network: Both Multi-NN and Multi-RNN decompose the conditional probability of both the level of abstraction $l$ and the lifted reward function $m$ given the natural language command $c$ as $\operatorname{Pr}(l, m \mid c)=\operatorname{Pr}(l \mid c) \cdot \operatorname{Pr}(m \mid l, c)$, allowing for the explicit calculation of the probability of each level of abstraction given the natural language command. As a result, both Multi-NN 


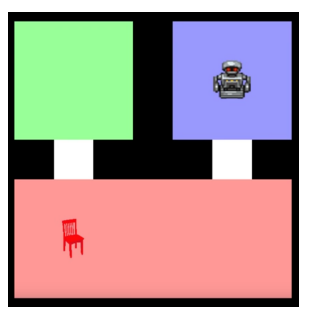

(a) A starting instance of the Cleanup World domain.

\begin{tabular}{|c|c|c|}
\hline Level & Example Command & Reward Function \\
\hline \multirow{2}{*}{$L_{0}$} & Turn and move one spot to the right. & goWest \\
\hline & Go three down, four over, two up. & agentInRoom agent0 roomlsGreen \\
\hline \multirow{2}{*}{$L_{1}$} & $\begin{array}{l}\text { Go to door, enter red room, } \\
\text { push chair to green room door. }\end{array}$ & blockInRegion block0 roomlsGreen \\
\hline & Go to the door then go into the red room. & agentInRegion agent0 roomlsRed \\
\hline$L_{2}$ & $\begin{array}{l}\text { Go to the green room. } \\
\text { Bring the chair to the blue room. }\end{array}$ & $\begin{array}{l}\text { agentInRegion agent0 roomlsGreen } \\
\text { blockInRegion block0 roomlsBlue }\end{array}$ \\
\hline
\end{tabular}

(b) Example commands and corresponding reward functions.

Fig. 3: Amazon Mechanical Turk (AMT) dataset domain and examples.

and Multi-RNN create separate sets of parameters for each of the separate outputs, i.e. separate parameters for each level of abstraction in the underlying hierarchical planner.

Alternatively, we can directly estimate the joint probability $\operatorname{Pr}(l, m \mid c)$. To do so, we propose a different type of RNN model that takes in a natural language command as a sequence of words $s$ (as in Multi-RNN), and directly outputs the joint probability of each tuple $(l, m)$, where $l$ denotes the level of abstraction, and $m$ denotes the lifted reward function at the given level. The Single-RNN objective is to find $\hat{\theta}$ such that:

$$
\hat{\theta}=\arg \max _{\theta} \sum_{(n, l, m)} \log \operatorname{Pr}(l, m \mid s, \theta)
$$

With this Single-RNN model, we are able to significantly improve model efficiency compared to the Multi-RNN model, as all levels of abstraction share a single set of parameters. Furthermore, removing the explicit calculation of the level selection probabilities allows for the possibility of positive information transfer between levels of abstraction, which is not necessarily possible with the previous models.

The Single-RNN architecture is shown in Fig. 2c. We use a single-output RNN, similar to the Multi-RNN architecture, with the key difference being that there is only a single output, with each element of the final output vector corresponding to the probability of each tuple of levels of abstraction and reward functions $(l, m)$ given the natural language command $c$.

To train the model, we minimize the cross-entropy loss of the joint probability term in Eqn. 6 Training hyperparameters are identical to Multi-RNN, and dropout is applied to the initial embedding layer and the penultimate hidden layer.

\section{Grounding Module}

In all of our models, the inferred lifted reward function template must be binded to environment-specific variables. The grounding module maps the lifted reward function to a grounded one that can be passed to an MDP planner. In our evaluation domain (see Fig. 11, it is sufficient for our grounding module to be a lookup table that maps specific environment constraints to object ID tokens. In domains with ambiguous constraints (e.g. a "chair" argument where multiple chairs exist), a more complex grounding module could be substituted. For instance, Artzi and Zettlemoyer [2] present a model for executing lambda-calculus expressions generated by a combinatory categorial grammar (CCG) semantic parser, which grounds ambiguous predicates and nested arguments.

\section{Evaluation}

Our evaluation tests the hypothesis that hierarchical structure improves the speed and accuracy of language grounding at multiple levels of abstraction. We measure grounding accuracy and planning speed in simulation with a corpus-based evaluation, and demonstrate our system on a Turtlebot robot.

\section{A. Mobile-Manipulation Robot Domain}

The Cleanup World domain [18, 20], illustrated in Fig. 3a is a mobile-manipulator robot domain that is partitioned into rooms (denoted by unique colors) with open doors. Each room may contain some number of objects which can be moved (pushed) by the robot. This problem is modeled after a mobile robot that moves objects around, analogous to a robotic forklift operating in a warehouse or a pick-and-place robot in a home environment. We use an AMDP from Gopalan et al. [14] for the Cleanup World domain, which imposes a three-level abstraction hierarchy for planning.

The combinatorially large state space of Cleanup World simulates real-world complexity and is ideal for exploiting abstractions. At the lowest level of abstraction $L_{0}$, the (primitive) action set available to the robot agent consists of north, south, east, and west actions. Users directing the robot at this level of granularity must specify lengthy step-by-step instructions for the robot to execute. At the next level of abstraction $L_{1}$, the state space of Cleanup World only consists of rooms and doors. The robot's position is solely defined by the region (i.e. room or door) it resides in. Abstracted actions are subroutines for moving either the robot or a specific block to a room or door. It is impossible to transition between rooms without first transitioning through a door, and it is only possible to transition between adjacent regions; any language guiding the robot at $L_{1}$ must adhere to these dynamics. Finally, the highest level of abstraction, $L_{2}$, removes the concept of doors, leaving only rooms as regions; all $L_{1}$ transition dynamics still hold, including adjacency constraints. Subroutines exist for moving either the robot or a block between connected rooms. The full space of subroutines at all levels and their corresponding propositional functions are defined by [14]. Fig. 3b shows a few collected sample commands at each level and the corresponding level-specific AMDP reward function.

\section{B. Procedure}

We conducted an Amazon Mechanical Turk (AMT) user study to collect natural language samples at various levels of 


\begin{tabular}{lrrr}
\hline & Evaluated $L_{0}$ & Evaluated $L_{1}$ & Evaluated $L_{2}$ \\
\hline Trained $L_{0}$ & $\mathbf{2 1 . 6 1 \%}$ & $\mathbf{1 7 . 2 0 \%}$ & $21.87 \%$ \\
Trained $L_{1}$ & $9.83 \%$ & $10.23 \%$ & $13.90 \%$ \\
Trained $L_{2}$ & $14.94 \%$ & $12.84 \%$ & $\mathbf{3 1 . 4 9 \%}$ \\
\hline
\end{tabular}

(a) IBM2 Reward Grounding Baselines

\begin{tabular}{lrrr}
\hline & Evaluated $L_{0}$ & Evaluated $L_{1}$ & Evaluated $L_{2}$ \\
\hline Trained $L_{0}$ & $\mathbf{7 7 . 6 7 \%}$ & $28.05 \%$ & $23.26 \%$ \\
Trained $L_{1}$ & $32.79 \%$ & $\mathbf{8 2 . 9 9 \%}$ & $74.65 \%$ \\
Trained $L_{2}$ & $14.19 \%$ & $58.62 \%$ & $\mathbf{8 7 . 9 1 \%}$ \\
\hline
\end{tabular}

(b) Single-RNN Reward Grounding Baselines

Fig. 4: Task grounding accuracy (averaged over 5 trials) when training IBM2 and Single-RNN models on a single level of abstraction, then evaluating commands from alternate levels. This is similar to the MacGlashan et al. [20] results, as we see that without accounting for abstractions in language, there is a noticeable effect on grounding accuracy.

\begin{tabular}{lcc}
\hline & Level Selection & Reward Grounding \\
\hline IBM2 & $79.87 \%$ & $27.26 \%$ \\
Multi-NN & $93.51 \%$ & $36.05 \%$ \\
Multi-RNN & $95.71 \%$ & $80.11 \%$ \\
Single-RNN & $\mathbf{9 5 . 9 1 \%}$ & $\mathbf{8 0 . 4 6} \%$ \\
\hline
\end{tabular}

Fig. 5: Accuracy of 10-Fold Cross Validation (averaged over 3 runs) for each of the models on the AMT Dataset.

abstraction in Cleanup World. Annotators were shown video demonstrations of ten tasks, always starting from the state shown in Fig. 3a For each task, users provided a command that they would give to a robot, to perform the action they saw in the video, while constraining their language to adhere to one of three possible levels in a designated abstraction hierarchy: fine-grained, medium, and coarse. This data provided multiple parallel corpora for the machine translation problem of task grounding. We measured our system's performance by passing each command to the language grounding system and assessing whether it inferred both the correct level of abstraction and the reward function. We also recorded the response time of the system, measuring from when the command was issued to the language model to when the (simulated) robot would have started moving. Accuracy values were computed using the mean of multiple trials of ten-fold cross validation. The space of possible tasks included moving a single step as well as navigating to a particular room, taking a particular object to a designated room, and all combinations thereof.

Unlike MacGlashan et al. [20], the demonstrations shown were not only limited to simple robot navigation and object placement tasks, but also included composite tasks (e.g. "Go to the red room, take the red chair to the green room, go back to the red room, and return to the blue room"). Commands reflecting a clear misunderstanding of the presented task, e.g. "please robot", were removed from the dataset. Such removals were rare; we removed fewer than 30 commands for this reason, giving a total of 3047 commands. Per level, there were $1309 L_{0}$ commands, $872 L_{1}$ commands, and $866 L_{2}$ commands. The $L_{0}$ corpus included more commands since the tasks of moving the robot one unit in each of the four cardinal directions do not translate to higher levels of abstraction.

\section{Robot Task Grounding}

We present the baseline task grounding accuracies in Fig. 4 to demonstrate the importance of inferring the latent abstraction level in language. We simulate the effect of an oracle that partitions all of the collected AMT commands into separate corpora according to the specificity of each command. For this experiment, any $L_{0}$ commands that did not exist at all levels of the Cleanup World hierarchy were omitted, resulting in a condensed $L_{0}$ dataset of 869 commands. We trained multiple IBM2 and Single-RNN models using data from one distinct level and then evaluated using data from a separate level. Training a model at a particular level of abstraction includes grounding solely to the reward functions that exist at that same level. Reward functions at the evaluation level were mapped to the equivalent reward functions at the training level (e.g. $L_{1}$ agentInRegion to $L_{0}$ agentInRoom). Entries along the diagonal represent the average task grounding accuracy for multiple, random 90-10 splits of the data at the given level. Otherwise, evaluation checked for the correct grounding of the command to a reward function at the training level equivalent to the true reward function at the alternate evaluation level.

Task grounding scores are uniformly quite poor for IBM2; however, IBM2 models trained using $L_{0}$ and $L_{2}$ data respectively result in models that substantially outperform those trained on alternate levels of data. It is also apparent that an IBM2 model trained on $L_{1}$ data fails to identify the features of the level. We speculate that this is caused, in part, by high variance among the language commands collected at $L_{1}$ as well as the large number of overlapping, repetitive tokens that are needed for generating a valid machine language instance at $L_{1}$. While these models are worse than what MacGlashan et al. [20] observed, we note that we do not utilize a task or behavior model. It follows that integrating one or both of these components would only help prune the task grounding space of highly improbable tasks and improve our performance.

Conversely, Single-RNN shows the expected maximization along diagonal entries that comes from training and evaluating on data at the same level of abstraction. These show that a model limited to a single level of language abstraction is not flexible enough to deal with the full scope of possible commands. Additionally, Single-RNN demonstrates more robust task grounding than statistical machine translation.

The task grounding and level inference scores for the models in Sec. IV are shown in Fig. 5. Attempting to embed the latent abstraction level within the machine language of IBM2 results in weak level inference. Furthermore, grounding accuracy falls even further due to sparse alignments and the sharing of tokens between tasks in machine language (e.g. agentInRoom agent0 room 1 at $L_{0}$ and agentInRegion agent0 room 1 at $L_{1}$ ). The fastest of all the neural models, and 
the one with the fewest number of parameters overall, MultiNN shows notable improvement in level inference over the IBM2; however, task grounding performance still suffers, as the bag-of-words representation fails to capture the sequential word dependencies critical to the intent of each command. Multi-RNN again improves upon level prediction accuracy and leverages the high-dimensional representation learned by initial RNN layer to train reliable grounding models specific to each level of abstraction. Finally, Single-RNN has near-perfect level prediction and demonstrates the successful learning of abstraction level as a latent feature within the neural model. By not using an oracle for level inference, there is a slight loss in performance compared to the results obtained in Fig. 4b, however, we still see improved grounding performance over Multi-RNN that can be attributed to the full sharing of parameters across all training samples allowing for positive information transfer between abstraction levels.

\section{Robot Response Time}

Fast response times are important for fluid human-robot interaction, so we assessed the time it would take a robot to respond to natural language commands in our corpus. We measured the time it takes for the system to process a natural language command, map it to a reward function, and then solve the resulting MDP to yield a policy so that the simulated robot would start moving. We used Single-RNN for inference since it was the most accurate grounding model, and only correctly grounded instances were evaluated, so our results are for 2634 of 3047 commands that Single-RNN got correct.

We compared three different planners to solve the MDP:

- BASE: A state-of-the-art flat (non-hierarchical) planner, bounded real-time dynamic programming (BRTDP [24]).

- AMDP: A hierarchical planner for MDPs [14]. At the primitive level of the hierarchy $\left(L_{0}\right), \mathbf{A M D P}$ also requires a flat planner; we use BASE to allow for comparable planning times. Because the subtasks have no compositional structure, a Manhattan-distance heuristic can be used at $L_{0}$. While BASE technically allows for heuristics, distance-based heuristics are unsuitable for the composite tasks in our dataset. This illustrates another benefit of using hierarchies: to decompose composite tasks into subtasks that are amenable to better heuristics.

- NH (No Heuristic): Identical to AMDP, but without the heuristic as a fair comparison against BASE.

We hypothesize NH is faster than BASE (due to use of hierarchy), but not as fast as AMDP (due to lack of heuristics).

Since the actual planning times depend heavily on the actual task being grounded (ranging from $5 \mathrm{~ms}$ for goNorth to 180 s for some high-level commands), we instead evaluate the relative times used between different planning approaches. Fig. 6a shows the results for all 3 pairs of planners. For example, the left-most column shows $\frac{\text { AMDP time }}{\text { BASE time }}$ the fact that most results were less than 1 indicates that AMDP usually outperforms BASE. Using Wilcoxon signed-rank tests, we find that each approach in the numerator is significantly faster $\left(p<10^{-40}\right)$ than the one in the denominator, i.e. AMDP is

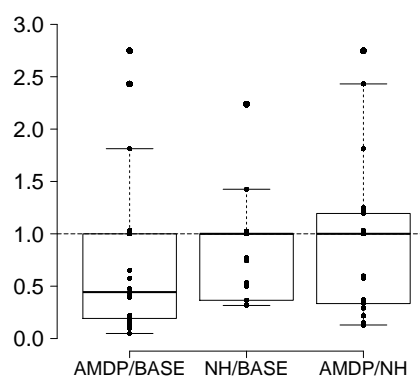

(a) Regular domain ( $2^{14}$ states)

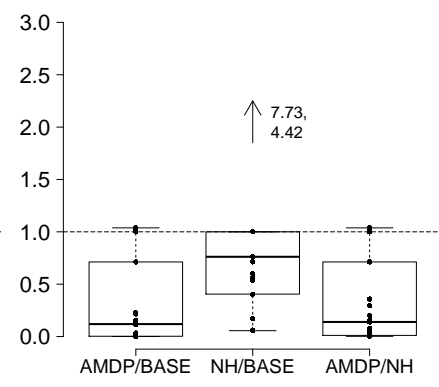

(b) Large domain ( $2^{18}$ states)
Fig. 6: Relative inference + planning times for different planning approaches on the same correctly grounded AMT commands. For each method pair, values less than 1 indicate the method on the numerator (left of '/') is better. Each data point is an average of 1000 planning trials.

faster than NH, which is in turn faster than BASE; this is consistent with our hypothesis. Comparing AMDP to BASE, we find that AMDP is twice as fast in over half the cases, 4 times as fast in a quarter of the cases, and can reach 20 times speedup. However, AMDP is also slower than BASE on $23 \%$ of the cases; of these, half are within $5 \%$ of BASE, but the other half is up to 3 times slower. Inspecting these cases suggests that the slowdown is due to overhead from instantiating multiple planning tasks in the hierarchy; this overhead is especially prominent in relatively small domains like Cleanup World. Note that in the worst case this is less than a 2 s absolute time difference.

From a computational standpoint, the primary advantage of hierarchy is space/time abstraction. To illustrate the potential benefit of using hierarchical planners in larger domains, we doubled the size of the original Cleanup domain and ran the same experiments. Ideally, this should have no effect on $L_{1}$ and $L_{2}$ tasks, since these tasks are agnostic to the discretization of the world. The results are shown in Fig. 6b. which again are consistent with our hypothesis. Somewhat surprisingly though, while NH still outperforms BASE $\left(p<10^{-150}\right)$, it was much less efficient than AMDP, which shows that the hierarchy itself was insufficient; the heuristic also plays an important role. Additionally, NH suffered from two outliers, where the planning problem became more complex because the solution was constrained to conform to the hierarchy; this is a well-known tradeoff in hierarchical planning [11]. The use of heuristics in AMDP mitigated this issue. AMDP times almost stayed the same compared to the regular domain, hence outperforming BASE and NH $\left(p<10^{-200}\right)$. The larger domain size also reduced the effect of hierarchical planning overhead: AMDP was only slower than BASE in $10 \%$ of the cases, all within $<4 \%$ of the time it took for BASE. Comparing AMDP to BASE, we find that AMDP is 8 times as fast in over half the cases, 100 times as fast in a quarter of the cases, and can reach up to 3 orders of magnitude in speedup. In absolute time, AMDP took $<1$ s on $90 \%$ of the tasks; in contrast, BASE takes $>20$ s on half the tasks. 


\section{E. Robot Demonstration}

Using the trained grounding model and the corresponding AMDP hierarchy, we tested with a Turtlebot on a smallscale version of the Cleanup World domain. To accommodate the continuous action space of the Turtlebot, the low-level, primitive actions at $L_{0}$ of the AMDP were swapped out for move forward, backward, and bidirectional rotation actions; all other levels of the AMDP remained unchanged. The low level commands used closed loop control policies, which were sent to the robot using the Robot Operating System [29]. Spoken commands were provided by an expert human user instructing the robot to navigate from one room to another. These verbal commands were converted from speech to text using Google's Speech API [1] before being grounded with the trained SingleRNN model. The resulting grounding, with both the AMDP hierarchy level and reward function, fed directly into the AMDP planner resulting in almost-instantaneous planning and execution. Numerous commands ranging from the low-level "Go north" all the way to the high-level "Take the block to the green room" were planned and executed using the AMDP with imperceivable delays after the conversion from speech to text. A video demonstration of the end-to-end system is available online: https://youtu.be/9bU2oE5RtvU

\section{DISCUSSION}

Overall, our best grounding model, Single-RNN, performed very well, correctly grounding commands much of the time; however, it still experienced errors. At the lowest level of abstraction, the model experienced some confusion between robot navigation (agentlnRoom) and object manipulation (blockInRoom) tasks. In the dataset, some users explicitly mention the desired object in object manipulation tasks while others did not; without explicit mention of the object, these commands were almost identical to those instructing the robot to navigate to a particular room. For example, one command that was correctly identified as instructing the robot to take the chair to the green room in Fig. 3a is "Go down...west until you hit the chair, push chair north..." A misclassified command for the same task was "Go south...west...north..." These commands ask for the same directions with the same amount of repetition (omitted) but only one mentions the object of interest allowing for the correct grounding. Overall, $83.3 \%$ of green room navigation tasks were grounded correctly while $16.7 \%$ were mistaken for green room object manipulation tasks.

Another source of error involved an interpretation issue in the video demonstrations presented to users. The robot agent shown to users as in Fig. 3a faces south and this orientation was assumed by the majority of users; however, some users referred to this direction as north (in the perspective of the robot agent). This confusion led to some errors in the grounding of commands instructing the robot to move a single step in one of the four cardinal directions. Logically, these conflicts in language caused errors for each of the cardinal directions as $31.25 \%$ of north commands were classified as south and $15 \%$ of east commands were labeled as west.
Finally, there were various forms of human error throughout the collected data. In many cases, users committed typos that actually affected the grounding result (e.g. asking the robot to take the chair back to the green room instead of the observed blue room). For some tasks, users often demonstrated some difficulty understanding the abstraction hierarchy described to them resulting in commands that partially belong to a different level of abstraction than what was requested. In order to avoid embedding a strong prior or limiting the natural variation of the data, no preprocessing was performed in an attempt to correct or remove these commands. A stronger data collection approach might involve adding a human validation step and asking separate users to verify that the supplied commands do translate back to the original video demonstrations under the given language constraints as in MacMahon et al. [21].

\section{CONCLUSION}

We presented a system for interpreting and grounding natural language commands to a mobile-manipulator robot at multiple levels of abstraction. To our knowledge, our system is not only the first work to ground language at multiple levels of abstraction, but also the first to use deep neural networks for language grounding on robots. Our proposed languagegrounding models significantly outperform the previous stateof-the-art method for mapping natural language commands to reward functions. By explicitly considering the level of abstraction, our system can interpret a much wider range of natural language commands, as well as leverage an existing hierarchical planner for efficient planning and execution of robot tasks. Finally, our Turtlebot evaluation demonstrates that this system works well in real-world environments and is an encouraging step towards seamless human-robot interaction.

To achieve natural interaction with humans, robots must be able to interpret all possible natural language input. Therefore, we must weaken the constraints and assumptions we place on input from users. To this end, we plan to extend our proposed models to handle natural language commands specified at a mixture of abstraction levels. More generally, we should not allow an existing planning abstraction hierarchy to constrain our interpretation of language. In contrast, we can use the space of user inputs to inform the learning of appropriate abstraction hierarchies, aiming to find structures that both match user language and are efficient to plan with.

We envision that our system is applicable to a large variety of real-world scenarios, particularly in environments where multiple levels of abstraction naturally occur, such as in surgical, manufacturing, and household robotics.

\section{ACKNOWLEDGEMENTS}

This work is supported by the National Science Foundation under grant number IIS-1637614, the US Army/DARPA under grant number W911NF-15-1-0503, and the National Aeronautics and Space Administration under grant number NNX16AR61G.

Lawson L.S. Wong was supported by a Croucher Foundation Fellowship. 


\section{REFERENCES}

[1] Google Speech API. https://cloud.google.com/speech/, 2017. Accessed: 2017-01-30.

[2] Yoav Artzi and Luke Zettlemoyer. Weakly supervized learning of semantic parsers for mapping instructions to actions. In Annual Meeting of the Association for Computational Linguistics, 2013.

[3] Richard Bellman. Dynamic Programming. Princeton University Press, Princeton, NJ, USA, 1 edition, 1957.

[4] Yoshua Bengio, Réjean Ducharme, Pascal Vincent, and Christian Janvin. A neural probabilistic language model. Journal of Machine Learning Research, 3:1137-1155, 2000.

[5] Daniel J. Brooks, Constantine Lignos, Cameron Finucane, Mikhail S. Medvedev, Ian Perera, Vasumathi Raman, Hadas Kress-Gazit, Mitch Marcus, and Holly A. Yanco. Make it so: Continuous, flexible natural language interaction with an autonomous robot. In AAAI Conference on Artificial Intelligence Workshop on Grounding Language for Physical Systems, 2012.

[6] Peter F. Brown, John Cocke, Stephen Della Pietra, Vincent J. Della Pietra, Frederick Jelinek, John D. Lafferty, Robert L. Mercer, and Paul S. Roossin. A statistical approach to machine translation. Computational Linguistics, 16:79-85, 1990.

[7] Peter F. Brown, Stephen Della Pietra, Vincent J. Della Pietra, and Robert L. Mercer. The mathematics of statistical machine translation: Parameter estimation. Computational Linguistics, 19:263-311, 1993.

[8] David L. Chen and Raymond J. Mooney. Learning to interpret natural language navigation instructions from observations. In AAAI Conference on Artificial Intelligence, 2011.

[9] Kyunghyun Cho, Bart van Merrienboer, Çaglar Gúlçehre, Dzmitry Bahdanau, Fethi Bougares, Holger Schwenk, and Yoshua Bengio. Learning phrase representations using rnn encoder-decoder for statistical machine translation. In Empirical Methods in Natural Language Processing, 2014.

[10] Junyoung Chung, Çaglar Gúlçehre, Kyunghyun Cho, and Yoshua Bengio. Empirical evaluation of gated recurrent neural networks on sequence modeling. CoRR, abs/1412.3555, 2014.

[11] Thomas G. Dieterrich. Hierarchical reinforcement learning with the MAXQ value function decomposition. Journal on Artificial Intelligence Research, 13:227-303, 2000.

[12] Carlos Diuk, Andre Cohen, and Michael L. Littman. An object-oriented representation for efficient reinforcement learning. In International Conference on Machine Learning, 2008.

[13] Juraj Dzifcak, Matthias Scheutz, Chitta Baral, and Paul W. Schermerhorn. What to do and how to do it: Translating natural language directives into temporal and dynamic logic representation for goal management and action execution. In IEEE International Conference on Robotics and Automation, 2009.

[14] Nakul Gopalan, Marie desJardins, Michael L. Littman, James MacGlashan, Shawn Squire, Stefanie Tellex, John Winder, and Lawson L.S. Wong. Planning with abstract Markov decision processes. In International Conference on Automated Planning and Scheduling, 2017.

[15] Thomas M. Howard, Stefanie Tellex, and Nicholas Roy. A natural language planner interface for mobile manipulators. In IEEE International Conference on Robotics and Automation, 2014.

[16] Mohit Iyyer, Varun Manjunatha, Jordan L. Boyd-Graber, and Hal Daumé. Deep unordered composition rivals syntactic methods for text classification. In Conference of the Association for Computational Linguistics, 2015.

[17] Nicholas K. Jong and Peter Stone. Hierarchical modelbased reinforcement learning: R-max + MAXQ. In International Conference on Machine Learning, 2008.

[18] Andreas Junghanns and Jonathan Schaeeer. Sokoban: a challenging single-agent search problem. In International Joint Conference on Artificial Intelligence Workshop on Using Games as an Experimental Testbed for AI Reasearch, 1997.

[19] Diederik P. Kingma and Jimmy Ba. Adam: A method for stochastic optimization. CoRR, abs/1412.6980, 2014.

[20] James MacGlashan, Monica Babeș-Vroman, Marie DesJardins, Michael L. Littman, Smaranda Muresan, Shawn Squire, Stefanie Tellex, Dilip Arumugam, and Lei Yang. Grounding English commands to reward functions. In Robotics: Science and Systems, 2015.

[21] Matt MacMahon, Brian Stankiewicz, and Benjamin Kuipers. Walk the talk: Connecting language, knowledge, and action in route instructions. In National Conference on Artificial Intelligence, 2006.

[22] Cynthia Matuszek, Evan Herbst, Luke Zettlemoyer, and Dieter Fox. Learning to parse natural language commands to a robot control system. In International Symposium on Experimental Robotics, 2012.

[23] Amy McGovern, Richard S. Sutton, and Andrew H Fagg. Roles of macro-actions in accelerating reinforcement learning. Grace Hopper Celebration of Women in Computing, 1317, 1997.

[24] H. Brendan McMahan, Maxim Likhachev, and Geoffrey J. Gordon. Bounded real-time dynamic programming: RTDP with monotone upper bounds and performance guarantees. In International Conference on Machine Learning, 2005.

[25] Tomas Mikolov, Martin Karafiát, Lukás Burget, Jan Cernocký, and Sanjeev Khudanpur. Recurrent neural network based language model. In Interspeech, 2010.

[26] Tomas Mikolov, Stefan Kombrink, Lukás Burget, Jan Cernocký, and Sanjeev Khudanpur. Extensions of recurrent neural network language model. In IEEE International Conference on Acoustics, Speech, and Signal Processing, 2011.

[27] Tomas Mikolov, Kai Chen, Gregory S. Corrado, and 
Jeffrey Dean. Efficient estimation of word representations in vector space. CoRR, abs/1301.3781, 2013.

[28] Rohan Paul, Jacob Arkin, Nicholas Roy, and Thomas M. Howard. Efficient grounding of abstract spatial concepts for natural language interaction with robot manipulators. In Robotics: Science and Systems, 2016.

[29] Morgan Quigley, Josh Faust, Tully Foote, and Jeremy Leibs. ROS: an open-source robot operating system. In IEEE International Conference on Robotics and Automation Workshop on Open Source Software, 2009.

[30] Vasumathi Raman and Hadas Kress-Gazit. Analyzing unsynthesizable specifications for high-level robot behavior using LTLMoP. In International Conference on Computer-Aided Verification, 2011.

[31] Nitish Srivastava, Geoffrey E. Hinton, Alex Krizhevsky, Ilya Sutskever, and Ruslan Salakhutdinov. Dropout: A simple way to prevent neural networks from overfitting. Journal of Machine Learning Research, 15:1929-1958, 2014.

[32] Ilya Sutskever, Oriol Vinyals, and Quoc V. Le. Sequence to sequence learning with neural networks. CoRR, abs/1409.3215, 2014.

[33] Richard S. Sutton, Doina Precup, and Satinder P. Singh. Between MDPs and semi-MDPs: A framework for temporal abstraction in reinforcement learning. Artificial Intelligence, 112:181-211, 1999.

[34] Stefanie Tellex, Thomas Kollar, Steven Dickerson, Matthew R. Walter, Ashis Gopal Banerjee, Seth Teller, and Nicholas Roy. Understanding natural language commands for robotic navigation and mobile manipulation. In AAAI Conference on Artificial Intelligence, 2011.

[35] Tatsuro Yamada, Shingo Murata, Hiroaki Arie, and Tetsuya Ogata. Dynamical linking of positive and negative sentences to goal-oriented robot behavior by hierarchical rnn. In International Conference on Artificial Neural Networks, 2016. 